\title{
The Role of the Exchange Interaction in the One-Dimensional $n$-Component Hubbard Model
}

\author{
E. Szirmai, Ö. Legeza And J. Sólyom \\ Research Institute for Solid State Physics and Optics \\ P.O. Box 49, H-1525 Budapest, Hungary
}

\begin{abstract}
The commensurate $p / q$-filled $n$-component Hubbard chain was investigated by bosonization and high-precision density-matrix renormalization-group analysis. It was found that depending on the relation between the number of components $n$, and the filling parameter $q$, the system shows metallic or insulating behavior, and for special fillings bond-ordered (dimerized, trimerized, tetramerized etc.) ground state develops in the insulating phase. A mean-field analysis shows that this bond ordering is a direct consequence of the spin-exchange interaction, which plays a crucial role in the one-parameter Hubbard model — not only for infinite Coulomb repulsion, but for intermediate values as well.
\end{abstract}

PACS numbers: 71.10.Fd

\section{Introduction}

Recent experimental results in ultracold gases in optical lattices may be simulated by multi-component fermionic systems in which spins can take more than two degrees of freedom [1]. A natural candidate for the description of such systems from the theoretical point of view is the $\mathrm{SU}(n)$-symmetric generalization of the standard SU(2) Hubbard model [2] which has been investigated intensively in the past by both analytic and numerical approaches $[1,3-10]$. In fact, this model may mimic strongly correlated electron systems where the orbital degrees of freedom of $d$ and $f$ electrons play important role and these extra degrees of freedom are taken into account by considering $n$-component fermions.

In this paper we will further analyze the physics of the $\mathrm{SU}(n)$ Hubbard model for commensurate fillings on the basis of our earlier works [6-8]. In Ref. [7] we have studied the occurrence of spatially nonuniform phases for special commensurate fillings and for different values of $n$, and the phase diagram of the model has been established. In the present paper, we will discuss the physics behind the phase diagram, and especially its implications for intermediate values of the Hubbard coupling $U$.

\section{Theory} form

The Hamiltonian of the model is usually written in the

$$
\begin{aligned}
\mathcal{H} & =\sum_{i=1}^{N}\left[-t \sum_{\sigma=1}^{n}\left(c_{i, \sigma}^{\dagger} c_{i+1, \sigma}+c_{i+1, \sigma}^{\dagger} c_{i, \sigma}\right)\right. \\
& \left.+\frac{U}{2} \sum_{\sigma, \sigma^{\prime}=1}^{n} n_{i, \sigma} n_{i, \sigma^{\prime}}\right]
\end{aligned}
$$

where $N$ is the number of sites in the chain. The oper- ator $c_{i, \sigma}^{\dagger}\left(c_{i, \sigma}\right)$ creates (annihilates) an electron at site $i$ with spin $\sigma$, where the spin index is allowed to take $n$ different values. $n_{i, \sigma}$ is the particle-number operator, $t$ is the hopping integral between nearest-neighbor sites, and $U$ is the strength of the on-site Coulomb repulsion. In what follows $t$ will be taken as the unit of energy.

It is well known that in the weak-coupling regime the half-filled $n$-component Hubbard model is an insulator with gapped charge and spin modes (for $n>2$ ), while in the large $U$ limit the system can be described - at least for even $n$ - by an effective Heisenberg model $[3,6]$. Away from half filling, based on leading-order renormalization group analysis, where the higher-order umklapp processes do not give contribution, one can find the Luttinger liquid behavior with gapless bosonic charge and spin modes. However, one can expect that these higher order processes become relevant for commensurate fillings and cannot be neglected. To see their effect, first we analyze how the spectrum of a Luttinger liquid is modified by these processes and then we study the occurrence of phases with spatial inhomogeneity for special fillings.

The well-known one-particle and particle-hole excitation spectrum of the two-component Luttinger liquid $[11,12]$ can be easily generalized for fermions with $n$ internal degrees of freedom

$$
E=\sum_{j} \hbar u_{j} \frac{2 \pi}{L}\left(n_{+}^{j}+n_{-}^{j}+\Delta_{+}^{j}+\Delta_{-}^{j}\right)
$$

where the momentum is quantized in units of $2 \pi / L$, and $n_{ \pm}^{j}$ are integers describing the particle-hole type bosonic excitations: the term $j=\mathrm{c}$ describes the excitations of the charge mode which is the symmetric combination of the bosonic phase fields $\phi_{\sigma}(x)$ of the corresponding fermion fields, $\phi_{\mathrm{c}}(x)=\frac{1}{\sqrt{n}} \sum_{\sigma=1}^{n} \phi_{\sigma}(x)$. The terms $j=m$ s describe the excitations of the $n-1$ 
spin modes which are independent antisymmetric combinations of the appropriate boson fields, $\phi_{m s}(x)=$ $[m(m+1)]^{-1 / 2}\left[\sum_{\sigma=1}^{m} \phi_{\sigma}(x)-m \phi_{m+1}(x)\right]$. In Eq. (2) $\Delta_{ \pm}^{j}$ corresponds to the one-particle excitations: $\Delta_{ \pm}^{j}=$ $\frac{1}{16}\left(\sqrt{K_{j}} J_{j} \pm \delta N_{j} / \sqrt{K_{j}}\right)^{2}$, where $\delta N_{j}$ is the change in the number of particles, and $J_{j}$ describes the current in the $j$-th channel generated by processes which break the chiral particle-number or spin conservation. Since the total momentum is given by

$$
P=\hbar k_{\mathrm{F}} J_{\mathrm{c}}+\sum_{j} \hbar \frac{2 \pi}{L}\left(n_{+}^{j}-n_{-}^{j}+\Delta_{+}^{j}-\Delta_{-}^{j}\right),
$$

and the charge current $J_{\mathrm{c}}$ is an even number due to the total particle-number conservation, in the thermodynamic limit soft modes appear not only at zero momentum but also at even integer multiples of $k_{\mathrm{F}}=\pi f$ for filling $f$.

For commensurate filling the higher-order umklapp processes are not irrelevant anymore, and they modify the Luttinger liquid spectrum. In the considered restricted Hilbert space (with low-energy states), the higher-order umklapp processes can be described only by multiparticle scatterings in fermion representation, and in these processes the number of scattered particles depends on the filling factor $f$. Namely, for $f=p / q$ filling, exactly $q$ particles take part in the leading-order multiparticle umklapp processes. Therefore, due to the Pauli principle these processes are forbidden for local interaction, if $q>n$, and the system remains Luttinger liquid. If, on the other hand, $q \leq n$, at least for strong interactions these processes are relevant, and their contribution has to be taken into account. The $q$-particle umklapp processes can be described in the terms of the bosonic fields as

$$
\begin{aligned}
& H_{\mathrm{U}}=g_{3} \int \mathrm{d} x \sum_{\left\{\sigma_{i}\right\}^{\prime}} \\
& \quad \times \cos \left(2\left(\phi_{\sigma_{1}}(x)+\ldots+\phi_{\sigma_{q}}(x)\right)\right) .
\end{aligned}
$$

Here $g_{3}$ is the coupling of $q$-particle umklapp processes and prime indicates that the summation over the spin indices gives contribution only for terms which contain phase fields with different spins. The other terms are forbidden by the Pauli principle. One can easily see from Eq. (4) that for $q=n$ the leading-order umklapp processes modify only the spectrum of the symmetric combination of the boson fields, which means that the charge excitations acquire a finite energy gap of order $g_{3}$, while the spin modes remain gapless. It is more interesting, as we will see, that if $q<n$, the leading-order umklapp scatterings couple the spin and charge modes, and due to this coupling the whole spectrum becomes gapped not only at zero momentum, but at $k^{*}=2 k_{\mathrm{F}}$, and integer multiples of $k^{*}$, too.

Considering the analytical predictions it is expected that the behavior of the system should be determined by the $k^{*}=2 k_{\mathrm{F}}$ modes. We have, therefore, studied the model numerically using the high-precision density-matrix renormalization group (DMRG) method [13] for several system sizes and values of $q$ and $n$ as a function of $U$. We have detected and located quantum phase transition points (QPTs) and determined the spatial characteristics of the ground state using various quantum information entropies [14-16]:

$$
s_{N}(l)=-\operatorname{Tr}\left(\rho_{N}(l) \ln \rho_{N}(l)\right),
$$

where a finite block of length $l$ of a long chain of $N$ sites is considered with the corresponding reduced subsystem density matrix $\rho_{N}(l)$. As has been shown before $[14,15]$, anomalies in the entropy functions or in their derivate signal QPTs, and peaks in the Fourier spectrum of $s_{N}(l)$ carry information about the position of soft modes (for critical models) or the spatial inhomogeneity of the ground state (for gapped systems) [16]. In the latter case the spatial modulation of the ground state can be a site- or a bond-centered density wave. A site-centered density wave would manifest itself in an oscillation of the entropy of single sites or in the local electron density. The existence of a bond-centered density wave can be demonstrated by studying the variation of the bond energy or the two-site entropy along the chain [15].

We have found that for models with $q \geq n$, for finite systems, the block entropy oscillates with a period determined by the filling, but all Fourier components except for $q=0$ disappear in the $N \rightarrow \infty$ limit. Therefore, the ground state of the system is spatially uniform.

A significantly different behavior has been found for systems with $q<n$. The block entropy function saturates beyond some system size [7] for finite $U$ values, indicating that the corresponding models are fully gapped [17]. In fact, gap opens in the spectrum of all modes for $U>0$ [8]. Even more interestingly, the translational symmetry of the Hamiltonian is broken and a spatially nonuniform ground state emerges whose periodicity depends on the filling. Since all Fourier components of the site entropy and local charge density have been found to vanish for long chains, the ground state is a bond-centered density wave. Therefore, we have identified bond-ordered dimerized, trimerized or tetramerized phases depending on the filling.

With the aim to interpret this result, the model was further studied analytically. However, starting with the Hamiltonian given in Eq. (1), we could not find any method that could reproduce the spatially nonuniform phases obtained numerically. On the other hand, we know that in the strong-coupling limit the Hubbard model is equivalent to the Heisenberg model with an effective antiferromagnetic exchange, and also that the one-dimensional Heisenberg model exhibits spin-Peierls instability. The spin chain dimerizes spontaneously when the exchange coupling depends on the distance between the neighbors. If the Hubbard model is extended with the Heisenberg term, the $J$ coupling might have a similar effect leading to the spatially nonuniform phases.

In order to analyze the possibility of bond ordering, we have taken a more general model, the HubbardHeisenberg model [3], which contains antiferromagnetic nearest-neighbor spin-exchange $J$, in addition to the 
on-site Coulomb repulsion $U$. The Hubbard-Heisenberg Hamiltonian is

$$
\begin{aligned}
\mathcal{H} & =\sum_{i=1}^{N}\left[-t \sum_{\sigma=1}^{n}\left(c_{i, \sigma}^{\dagger} c_{i+1, \sigma}+\text { h.c. }\right)\right. \\
& \left.+\frac{U}{2} \sum_{\sigma, \sigma^{\prime}=1}^{n} n_{i, \sigma} n_{i, \sigma^{\prime}}+J \boldsymbol{S}_{i} \boldsymbol{S}_{i+1}\right]
\end{aligned}
$$

where we use the same notations as in Eq. (1), and $\boldsymbol{S}_{i}$ is the $\mathrm{SU}(n)$ spin operator. Our mean-field analysis was based on a large- $n$ limit calculations and it was restricted to one-third-filled system, with $n$ an integer multiple of 3 as the special case of the filling $f=p / q, q<n$. We have found that the spatially homogeneous phase is not stable, the spatial periodicity changes for arbitrary small positive value of $J$ : it seems that the Hubbard model is unstable against the antiferromagnetic nearest-neighbor Heisenberg exchange, independently of the value of the on-site Coulomb interaction.

\section{Conclusions}

We conclude that the exchange correlations that are present in the Hubbard model for any value of $U$, become relevant in the intermediate-coupling regime, too, and they are responsible for the spatial distortion. Our results also show that these correlations are difficult to treat analytically in that coupling regime where neither the weaknor the strong-coupling methods work resonably. Therefore, in order to take the effects of these processes into account, it is sensible to add a Heisenberg-like nearest-neighbor exchange to the Hamiltonian of the Hubbard model. The strongest argument for the relevance of a Heisenberg-like exchange in the Hubbard model would be a non-perturbative real-space renormalization group analysis which could be the object of a future work.

\section{Acknowledgments}

This research was supported by the Hungarian Research Fund (OTKA) grants No. K 68340, F 046356 and NF 61726 and the János Bolyai Research Fund. The authors acknowledge computational support from Dynaflex Ltd. under grant No. IgB-32.

\section{References}

[1] C. Honerkamp, W. Hofstetter, Phys. Rev. Lett. 92, 170403 (2004).

[2] J. Hubbard, Proc. R. Soc. A 276, 238 (1963); ibid. 277, 237 (1964); ibid. 281, 401 (1964); ibid. 285, 542 (1965).

[3] J.B. Marston, I. Affleck, Phys. Rev. B 39, 11538 (1989).

[4] R. Assaraf, P. Azaria, M. Caffarel, P. Lecheminant, Phys. Rev. B 60, 2299 (1999); R. Assaraf, P. Azaria, E. Boulat, M. Caffarel, P. Lecheminant, Phys. Rev. Lett. 93, 016407 (2004).

[5] F.F. Assaad, Phys. Rev. B 71, 075103 (2005).

[6] E. Szirmai, J. Sólyom, Phys. Rev. B 71, 205108 (2005); ibid. 74, 155110 (2006).

[7] E. Szirmai, Ö. Legeza, J. Sólyom, Phys. Rev. B 77, 045106 (2008).

[8] K. Buchta, Ö. Legeza, E. Szirmai, J. Sólyom, Phys. Rev. B 75, 155108 (2007).

[9] Á. Rapp, G. Zaránd, C. Honerkamp, W. Hofstetter, Phys. Rev. Lett. 98, 160405 (2007).

[10] J. Zhao, K. Ueda, X. Wang, arXiv:cond-mat/0702582.

[11] For a review of renormalization group method and bosonization see: J. Sólyom, Adv. Phys. 28, 201 (1979); A. Gogolin, A. Nersesyan, A. Tsvelik, Bosonization and Strongly Correlated Systems, Cambridge University Press, Cambridge 1998.

[12] F.D.M. Haldane, J. Phys. C 14, 2585 (1981).

[13] S.R. White, Phys. Rev. Lett. 69, 2863 (1992); Phys. Rev. B 48, 10345 (1993).

[14] J. Vidal, G. Palacios, R. Mosseri, Phys. Rev. A 69, 022107 (2004); J. Vidal, R. Mosseri, J. Dukelsky, ibid. 69, 054101 (2004).

[15] Ö. Legeza, J. Sólyom, Phys. Rev. Lett. 96, 116401 (2006).

[16] Ö. Legeza, J. Sólyom, L. Tincani, R.M. Noack, Phys. Rev. Lett. 99, 087203 (2007).

[17] G. Vidal, J.I. Latorre, E. Rico, A. Kitaev, Phys. Rev. Lett. 90, 227902 (2003). 